\title{
Evaluation of Geriatric Patients with Dry Mouth by Community Pharmacists and Use of Moisturizing Agents
}

\author{
Miyoshi Kawakami $^{* 1}$, Yoko Tashiro, Takahiro Oshima ${ }^{2}$, Ikuko Hashimoto ${ }^{2}$ and Yuji Yoshiyama ${ }^{1}$ \\ Laboratory of Community Pharmacy, Division of Clinical Pharmacy, \\ Research and Education Center for Clinical Pharmacy, School of Pharmacy, Kitasato University ${ }^{\text {, }}$ \\ Oshima Pharmacy ${ }^{2}$ \\ $\left[\begin{array}{l}\text { Received June 4, } 2012 \\ \text { Accepted August 14, } 2012\end{array}\right]$
}

Dry mouth is a common complaint among elderly people; it is often associated with risks of various disorders. Maintaining oral health helps maintain general health, which is important in aging populations. In Japan, many elderly people take prescription medications and visit community pharmacies. This study investigated the prevalence of geriatric patients complaining of dry mouth and its relationship with objective oral dryness measurements. We also investigated the efficacy of a moisturizing agent on oral dryness.

The subjects were 140 geriatric patients living in their homes and visiting community pharmacies regularly. Subjective complaints of dry mouth were determined by face-to-face interview and questionnaires. Objective oral dryness was measured using an oral wetness test, KISO-WeT. The efficacy of a moisturizing agent, KINUSUI ${ }^{\circledR}$, was investigated in the patients who had both subjective and objective dryness.

The mean age of the patients was 75.1 years. A total of $38.6 \%$ of patients complained of dry mouth; of them, $86.0 \%$ had objective oral dryness. Most (85.7\%) patients who used the moisturizing agent reported positive effects; objective oral dryness improved significantly as a result of using the agent $(P<0.05)$. Over $40 \%$ of users stated that they would continue using the moisturizing agent.

A substantial proportion of geriatric patients complained of dry mouth. The present results indicate that objective measurements of dryness are closely associated with subjective complaints. Pharmacists in community pharmacies are able to collect necessary information and offer moisturizing agents to benefit the oral health of geriatric patients.

Key words — dry mouth, geriatric patients, community pharmacy, moisturizing agent

\section{Introduction}

The Japanese population has aged rapidly in recent decades. In 2010, people 65 years old or older comprised $23.3 \%$ of the total population in Japan. Extending healthy life expectancy is important; however, it has become even more important to prevent disease, maintain health, and provide primary care. A revised system of preventionoriented care was developed in 2006 in light of the increasing number of people requiring longterm care. Furthermore, maintaining and improv- ing the oral health of the elderly is important. ${ }^{1-4)}$

Proper oral function involves saliva secretion, speaking, food intake, communication, etc. Maintaining oral function benefits not only physical health, but mental health as well. Saliva has various functions including aiding digestion, antibacterial effects, and maintaining bodily fluids.

Elderly people complaining of oral dryness, tongue pain, and taste disturbance due to diminished oral function often have serious symptoms that hinder their independence.

This study investigated the prevalence of sub-

* 5-9-1 Shirokane, Minato-ku, Tokyo, 108-8641 Japan 
jective complaints of dry mouth and whether they are correlated to objective measurements of oral dryness.

We also investigated the efficacy of a moisturizing agent. Hyaluronic acid is among the many moisturizing agents available in community pharmacies; it is a glycosaminoglycan and has excellent water retention properties. ${ }^{5)}$ It was selected for use in this study because it is a first-line moisturizing agent.

\section{Methods}

\section{Survey of the prevalence of subjective complaints of dry mouth and relation- ship with objective measurements of oral dryness}

This study was conducted at 2 community pharmacies in Kanagawa Prefecture (located south of Tokyo) in September and December 2010. The subjects were 140 geriatric patients: 56 men and 84 women $\geq 65$ years old with a mean age of 75.1 years living in their homes who regularly visited community pharmacies. We collected information about subjective complaints of dry mouth from all patients by face-to-face interviews and questionnaires. Oral dryness was measured objectively using an oral wetness test (KISOWeT, KISO Science Ltd., Yokohama, Japan) in 40 of the 140 patients.

This study was approved by the Kitasato University Kitasato Institute Hospital Research Ethics Committee. All patients were informed of the purpose of the study and provided written consent prior to participation.

Patients diagnosed with Sjögren syndrome or dry mouth due to temporary medicine use, or those who underwent radiation therapy or were currently receiving treatment for oral dryness
Table 1 Three questions about subjective complaints of dry mouth

1. Do you feel dry mouth?

2. Do you have a pain in your tongue, smarting pain?

3. Do you feel sticky paste in your mouth or is it not easy to talk?

were excluded.

Face-to-face interview and questionnaire regarding subjective complaints of dry mouth; community pharmacists asked 3 questions (Table 1). If the patient answered "yes" to even one question, the patient was recorded as positive for subjective complaints of dry mouth. To confirm whether a patient's subjective complaint of dry mouth was consistent with objective measurements of oral dryness, a saliva moisture test was performed using an oral wetness test, KISO-WeT. KISO-WeT is composed of an ultra-thin absorptive membranous filter material on top of a polyester film and measures the degree of oral moisture. The measurement site was the dorsal side of the tongue, $10 \mathrm{~mm}$ from the apex linguae. The examination paper was maintained vertically on the tongue for 10 seconds, and the height $(\mathrm{mm})$ of the scale that had been adsorbed was recorded. Patients were prohibited from eating and drinking water 30 minutes prior to measurement to standardize conditions. Tongue moistness was scored on a scale of 1-5: abundant (>5.0 mm), normal (3.0-4.9 mm), scarce (1.0-2.9 mm), dry (0.1-0.9 $\mathrm{mm})$, and very dry $(0 \mathrm{~mm})$ (Fig. 1).

\section{Hyaluronic acid-containing moisturiz- ing agent trial}

Eighteen geriatric patients ( 5 men, 13 women, mean age: 74.3 years) who had both subjective and objective oral dryness in the abovementioned study were enrolled in the moisturizing agent trial. The effectiveness of the moisturizing agent was 


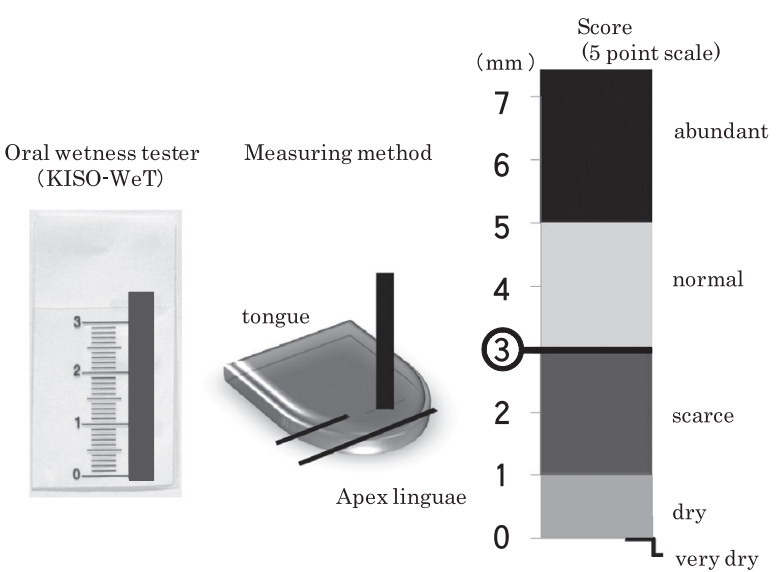

Fig. 1 Oral moisture test procedure and evaluation

determined according to the change in subjective feeling and objective saliva moisture after use. We used KINUSUI ${ }^{\circledR}$ (Mouthwash Spray, Seikagaku Corporation), which contains hyaluronic acid (moisturizing agent), purified water (solvent), xylitol (taste-masking agent), sodium benzoate, potassium sorbate (preservative), and sodium and disodium phosphate ( $\mathrm{pH}$ adjustment). Patients used the moisturizing agent at home until their next visit to the community pharmacy ( 2 weeks to 1 month). The moisturizing agent was administered in 4-5 sprays for a maximum of 5 times per day when patients complained of dry mouth. Patients answered questions regarding the effects of the moisturizing agent and desire to continue using the product during their subsequent visit to the community pharmacy.

We used the chi-square test to determine whether there were any gender differences regarding subjective complaints of dry mouth, Student's $t$-test to compare objective oral moisture between subjective and non-subjective complaints of dry mouth and paired $t$-tests to compare objective oral moisture before and after the use of the moisturizing agent. The level of statistical significance was set at $P<0.05$.

\section{Results}

\section{Prevalence of subjective complaints of dry mouth and relationship with objec- tive measurements of oral dryness}

The prevalence of subjective complaints of dry mouth was $38.6 \%$ (54/140), including 19 men and 35 women (mean age: $75.4 \pm 6.3$ years); there was no difference between men and women (Table 2). Forty patients participated in the objective measurement of oral dryness. Twenty-one patients ( 7 men, 14 women, mean age: $73.9 \pm 5.2$ years) had subjective complaints of dry mouth, whereas 19 (10 men, 9 women, mean age: 76.6 \pm 7.4 years) did not complain of dry mouth. The saliva moisture test results in the group $(n=21)$ with subjective complaints of dry mouth were as follows: abundant $4.8 \%(1 / 21)$, normal $9.5 \%$ (2/21), and dry $86.0 \%$ (18/21). Conversely, among the 19 patients without subjective complaints of dry mouth, $10.5 \%, 73.7 \%, 15.8 \%$ were abundant, normal, and dry, respectively. A total of $84.2 \%$ of patients who did not have subjective complaints of dry mouth had objectively normal or abundant test results (Table 3).

Table 2 Gender differences in subjective complaints of dry mouth

\begin{tabular}{lccc}
\hline \hline & $\begin{array}{c}\text { Total } \\
\mathrm{n}=140\end{array}$ & $\begin{array}{c}\text { Men } \\
\mathrm{n}=56\end{array}$ & $\begin{array}{c}\text { Women } \\
\mathrm{n}=84\end{array}$ \\
\hline $\begin{array}{l}\text { Subjective complaints } \\
\text { of dry mouth }\end{array}$ & $54(38.6 \%)$ & $19(33.9 \%)$ & $35(41.7 \%)$ \\
$\begin{array}{l}\text { Non-subjective complaints } \\
\text { of dry mouth }\end{array}$ & $86(61.4 \%)$ & $37(66.1 \%)$ & $49(58.3 \%)$ \\
\hline
\end{tabular}


Table 3 Saliva moisture test

\begin{tabular}{lccc}
\hline \hline & Abundant & Normal & Dry \\
\hline $\begin{array}{l}\text { Subjective complaints } \\
\text { of dry mouth }(\mathrm{n}=21)\end{array}$ & $1(4.8 \%)$ & $2(9.5 \%)$ & $18(86.0 \%)$ \\
$\begin{array}{l}\text { Non- subjective complaints } \\
\text { of dry mouth }(\mathrm{n}=19)\end{array}$ & $2(10.5 \%)$ & $14(73.7 \%)$ & $3(15.8 \%)$
\end{tabular}

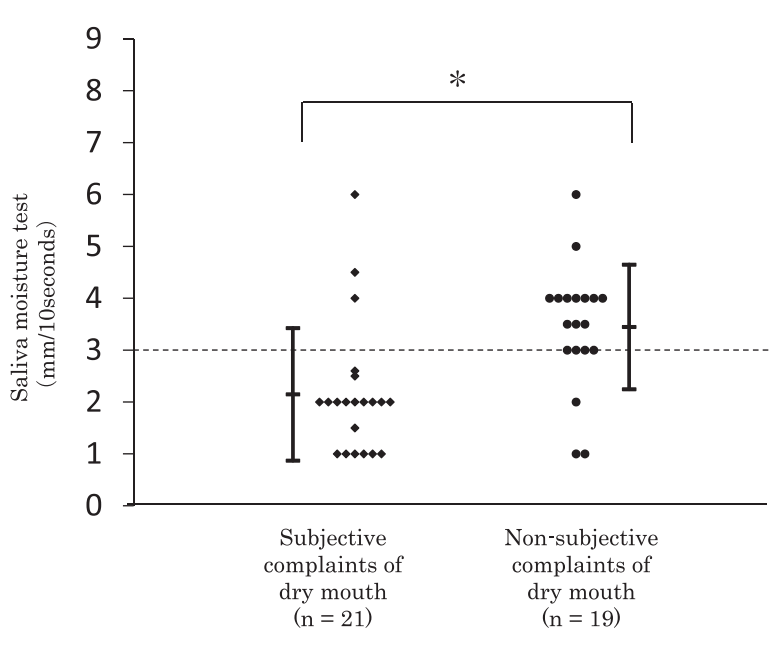

Fig. 2 Oral moisture of patients with subjective complaints of dry mouth and objective oral moisture findings ${ }^{*} P<0.05$ : Student's $t$-test

The group with subjective complaints of dry mouth had lower objective dryness scores than those of not complaining of dry mouth $(2.1 \pm 1.3$ vs $3.4 \pm 1.2 \mathrm{~mm}$, respectively) $(P<0.05)$ (Fig. 2$)$.

\section{Hyaluronic acid-containing moisturiz- ing agent trial}

In the 18 patients who had both subjective complaints of dry mouth and objective oral dryness, the degree of saliva moisture improved significantly from $1.7 \pm 0.6 \mathrm{~mm}$ before use to $3.4 \pm 1.5 \mathrm{~mm}$ after use $(P<0.05)($ Fig. 3). Fourteen of the 18 patients who had both subjective complaints of oral dryness and objective oral dryness answered the questionnaire when they returned to the community pharmacy. Regarding the feeling of oral moisture, $14.3 \%$ (2/14), $71.4 \%$ (10/14), and $14.3 \%(2 / 14)$ reported "very good," "somewhat good," and "not good," respectively (Fig. 4). A to-

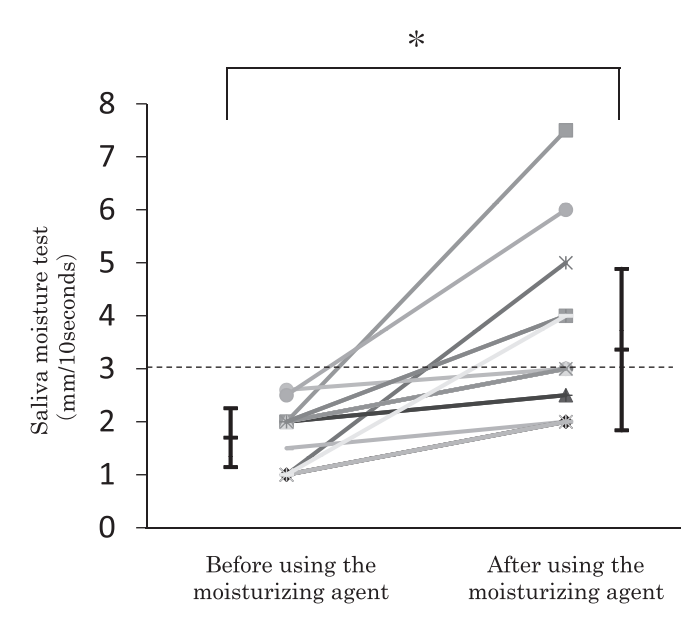

Fig. 3 Oral moisture before and after the use of the moisturizing agent $(n=18$, both subjective and objective oral dryness groups) ${ }^{*} P<0.05$ : paired $t$-test

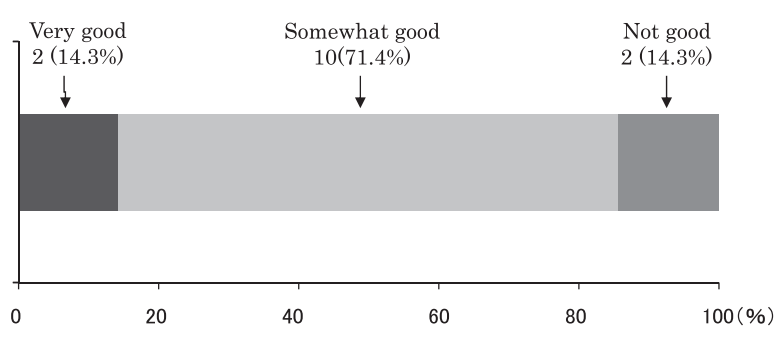

Fig. 4 Subjective feeling of oral moisture after moisturizing agent use

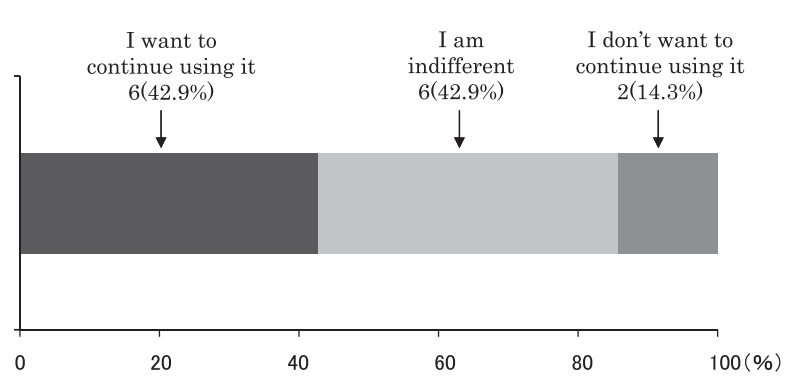

Fig. 5 Desire to continue using the moisturizing agent

tal of $85.7 \%$ reported some level of moisture after using the product. Regarding the patients' desire to continue using the moisturizing agent, 
$42.9 \%$ (6/14) wanted to continue using it, $42.9 \%$ (6/14) were indifferent, and $14.3 \%(2 / 12)$ did not want to continue using it (Fig. 5). The 2 patients who did not want to continue using the moisturizer reported that the agent did not give them a moist feeling in their mouth.

\section{Discussion}

Nearly $40 \%$ of geriatric patients who attended a community pharmacy to pick up their prescription medications had subjective complaints of dry mouth. The present results are consistent with those of previous studying indicating that 20 $39 \%$ of people $\geq 65$ years old complain of dry mouth. ${ }^{6-8)}$ This finding highlights the need to address dry mouth complaints in community pharmacies. In the present study, a saliva moisture test was performed prior to recommending dry mouth care solely on the basis of the history of a patient's subjective complaints of dry mouth to validate their complaints. The high percentage (86.0\%) of patients who both subjective complaints of dry mouth and objective dryness suggests that subjective complaints of dry mouth are a good indicator of objective oral dryness. In addition, $84.2 \%$ of geriatric patients who did not have complaints of dry mouth did not exhibit objective oral dryness. Therefore, the subjective symptom of dry mouth in this group of geriatric patients was closely associated with objective oral dryness measurements. Therefore, in community pharmacies, considering subjective dry mouth complaints without objectively measuring saliva moisture appears reasonable.

We also evaluated the effectiveness of a moisturizing agent available in community pharmacies. We used KINUSUI ${ }^{\circledR}$, a moisturizing agent that contains hyaluronic acid. Hyaluronic acid is glycosaminoglycan, which is composed of Dglucuronic acid and D- $N$-acetylglucosamine linked by alternating $\beta-1,4$ and $\beta-1,3$ glycoside bonds; it can have 25,000 disaccharide repeats." It has excellent water retention properties and is a first-line moisturizing agent.

In the both the subjective and objective evaluations of dry mouth symptoms, the moisturizing agent significantly improved oral dryness. Subjectively, $85.7 \%$ of patients reported improvement in dryness, and $42.9 \%$ reported that they wanted to continue using the moisturizing agent. Only the 2 patients who did not report improved moisture did not want to continue using it.

To our knowledge, this is the first investigation of oral dryness in independent geriatric patients who attend community pharmacies. The present results indicate that community pharmacists can identify the subjective symptom of dry mouth and make recommendations for preventive approaches in long-term care. Historically, community pharmacies only recommended oral moisturizing agents occasionally. However, the present results suggest a possible new role of community pharmacies in preventive approaches.

Dry mouth is a common complaint among the elderly. ${ }^{9,10)}$ The causes of dry mouth can be related to autoimmune diseases such as Sjögren's syndrome as well as irradiation treatment for oral neoplasms and the use of certain medications. ${ }^{10}$ Multiple medications for treating multiple disease states are thought to be related to dry mouth symptoms. Therefore, elderly people, who often take multiple medications, might report dry mouth symptoms depending on their medications. ${ }^{11)}$ Previous studies indicate that the prevalence of dry mouth in patients taking one or more medications is significantly higher than that in patients not taking any medications. ${ }^{12-14)}$ Although 
we did not specifically study this factor, all patients who had both subjective and objective oral dryness were taking at least one medication listing oral dryness as a potential side-effect. In the future, we will further examine the relationship between medicines and complaints of dry mouth.

Community pharmacists can identify and address dry mouth. In addition to the treatment of conditions, prevention, health maintenance, and primary care become more important in aging populations.

\section{References}

1) Ibayashi H, Fujino Y, Pham TM, Matsuda S, Intervention study of exercise program for oral function in healthy elderly people, Tohoku J Exp Med, 2008, 215, 237-245.

2) Appollonio I, Carabellese C, Frattola A, Trabucchi M, Dental status, quality of life, and mortality in an older community population: A multivariate approach, $J$ Am Geriatr Soc, 1997, 45, 1315-1323.

3) Yoneyama T, Yoshida M, Ohrui T, Mukaiyama H, Okamoto H, Hoshida K, Ihara S, Yanagisawa S, Ariumi S, Morita T, Mizuno Y, Ohsawa T, Akigawa Y, Hashimoto K, Sasaki H, Oral care reduces pneumonia in older patients in nursing homes, J Am Geriatr Soc, 2002, 50, 430-433.

4) Robbins J, Gangnon R, Theis S, Kays S, Hewitt A, Hind $\mathrm{J}$, The effects of lingual exercise on swallowing in older adults, J Am Geriatr Soc, 2005, 53, 14831489.
5) Higuchi Y, Ansai T, Awano S, Soh I, Yoshida A, Hamasaki T, Kakinoki Y, Takehara T, Salivary levels of hyaluronic acid in female patients with dry mouth compared with age-matched controls: a pilot study, Biomed Res, 2009, 30, 63-68.

6) Osterberg T, Landahl S, Hedegard B, Salivary flow, saliva $\mathrm{pH}$ and buffering capacity in 70-year-old men and women, J Oral Rehabil, 1984, 11, 157-170.

7) Gilbert GH, Heft MW, Duncan RP, Mouth dryness as reported by older Floridians, Community Dent Oral Epidemiol, 1993, 21, 390-397.

8) Thomson WM, Brown RH, Williams SM, Medication and perception of dry mouth in a population of institutionalized elderly people, $N Z$ Med J, 1993, 106, 219-221.

9) Närhi TO, Prevalence of subjective feelings of dry mouth, J Dent Res, 1994, 73, 20-25.

10) Loesche WJ, Bromberg J, Terpenning MS, Bretz EA, Dominguez BL, Grossman NS, Langmore SE, Xerostomia, xerigenic medications and food avoidances in selected geriatric groups, J Am Geriatr Soc, 1995, 43, 401-407.

11) Sreebny LM, Schwartz SS, A reference guide to drugs and dry mouth -2nd edition, Gerodontol, 1997, 14, 33-47.

12) Villa A, Abati S, Risk factors and symptoms associated with xerostomia: a cross-sectional study, Aust Dent J, 2011, 56, 290-295.

13) Field, EA, Fear S, Higham SM, Iewland RS, Rostron J, Willetts RM, Longman LP, Age and medication are significant risk factors for xerostomia in an English population, attending general dental practice, Gerodontol, 2001, 18, 21-24.

14) Wolff A, Zuk-Paz L, Kaplan I, Major salivary gland output differs between users and non-users of specific medication categories, Gerondontol, 2008, 25, 210-216. 